\title{
Performance of FSO Links under Exponentiated Weibull Turbulence Fading with Misalignment Errors
}

\author{
P. K. Sharma ${ }^{\dagger, 1}$, A. Bansal ${ }^{\ddagger}, 2$, P. Garg ${ }^{\ddagger, 3}$, T. A. Tsiftsis ${ }^{\dagger \dagger, 4}$ and R. Barrios ${ }^{\dagger \dagger, 5}$ \\ ${ }^{\dagger}$ Department of EECE, ITM University, Gurgaon, India \\ $¥$ Division of ECE, Netaji Subhas Institute of Technology, New Delhi, India \\ $\dagger \dagger$ Dept. of Electrical Engineering, Technological Educational Institute of Central Greece, Lamia, Greece \\ $\dagger \ddagger$ Institute of Communications and Navigation, German Aerospace Center (DLR), 82234 Wessling, Germany \\ prabhatmadhavec1@gmail.com ${ }^{1}$, bansal.ankur143@gmail.com², parul_saini@yahoo.co.in ${ }^{3}$, \\ tsiftsis@teilam.gr ${ }^{4}$, Ricardo.Barrios@dlr.de ${ }^{5}$
}

\begin{abstract}
The exponentiated Weibull (EW) distribution has been recently proposed for the modelling of free-space optical (FSO) links in the presence of finite sized receiver aperture. In this paper, the performance of FSO communication systems over EW is studied. Specifically, the probability density function (PDF) and cumulative distribution function (CDF) of the instantaneous signal-to-noise ratio (SNR), over EW turbulence fading, are studied. The derived statistics of the SNR is utilized to analyse the performance of an FSO communication system over a generalized communication environment with turbulence induced fading, misalignment errors and path loss. New expression for the outage probability is obtained, and exact expressions for the average bit error rate (BER) are derived for various binary modulation schemes. Finally, the obtained analytical results are verified via Monte Carlo simulations.
\end{abstract}

\section{INTRODUCTION}

Free-space optical (FSO) communication has emerged recently as an efficient solution to match the larger bandwidth and high data rates requirement of the upcoming wireless communication systems. However, the major challenge in establishing a wireless link at optical frequencies is atmospheric turbulence experienced by the laser beam when it propagates through the medium [1]. The atmospheric turbulence causes various perturbations in the propagating beam like scintillation which represents the random fluctuations in the beam irradiance, beam wander which stands for the random movement of the instantaneous centre of the beam at receiving aperture, and beam spreading that indicates the spreading beyond the diffraction limit of the beam radius [2].

The receivers in FSO systems generally have the point geometry which further worsens their performance in presence of the atmospheric turbulence. To improve the performance of a communication system in atmospheric turbulence, several techniques have been proposed in the literature [3]-[4]. The larger dimensions of the apertures in the receiver may help in collecting the higher irradiance flux, but it will place a hardware constraint on the receiver structures. Aperture averaging is one of the most widely used alternative technique due to its simplicity and lower cost. In aperture averaging, to mitigate the adverse effect of the atmospheric turbulence induced fading, a collecting aperture is placed at the end of the FSO link in the receiver side. The placement of the collecting aperture integrates the more light in the receiver plane and thus a greater portion of the incoming wavefront can be concentrated into the photodetector of the point receiver.

The characterization of the atmospheric turbulence induced fading is an important step in the performance analysis of an FSO communication systems. The existing channel models such as log-normal and Gamma-Gamma can not be used in all turbulence situations, and even sometimes they do not provide a good fit for experimental and simulated data [2], [5]. Recently, Barrios et. al. in [6], [7] proposed a new distribution, known as exponentiated-Weibull (EW) distribution, to model the atmospheric turbulence induced fluctuations in the irradiance received at the finite size aperture-averaged receiver. The EW distribution captures the effect of apertureaveraging through its constituent parameters as these parameters depend on the scintillation index of the received irradiance.

The performance of the FSO communication system over EW channels has been analysed in [8]-[12]. The approximate expressions for the bit error rate (BER) are derived in [8]-[11]. In [8], [11], the BER is obtained using GaussLaguerre quadrature rule, while Gauss-Hermite quadrature approximation is used in [9]. The average capacity of the optical wireless communication systems over EW distribution turbulence channels is derived in [12]. However, the analysis in all these works utilized the probability density function (PDF) of the fading channel, and none of them derived and utilized the PDF of the signal-to-noise ratio (SNR). The statistical analysis through SNR based approach is less complex and more general as can be extended to different modulation techniques directly. So in this paper we, first derive the distribution of the instantaneous SNR, and apply the derived statistics to analyze the outage and error performance of the FSO communication systems. Specifically, expression for the outage probability using the cumulative distribution function (CDF) of the instantaneous SNR is obtained. Finally, the expression for the average bit error rate (BER) is derived, which is applicable for various binary modulation schemes such as coherent binary frequency shift keying (BFSK), non- 
coherent BFSK, coherent binary phase shift keying (BPSK), and differential BPSK.

The rest of this paper is organized as follows: In section II the detailed description of channel model is given. The PDF and CDF of the instantaneous SNR are derived in section III. Performance analysis metrics such as the outage probability and average BER are presented in Section IV. Section V details the numerical results and conclusions are given in section VI.

\section{Channel Model}

We consider the composite model for the channel between the source and the destination, represented by the channel coefficient $h$. It is composed of atmospheric turbulence induced fading represented by the coefficient $h_{I}$, the misalignment fading denoted as $h_{m}$, and distance dependent path loss $h_{\ell}$. Thus composite channel coefficient can be given as

$$
h=h_{I} h_{m} h_{\ell} .
$$

The atmospheric turbulence induced fading is modelled under EW distribution. The PDF of the coefficient $h_{I}\left(h_{I}>\right.$ $0)$, is [6]

$$
f_{h_{I}}\left(h_{I}\right)=\frac{\alpha \beta}{\eta}\left(\frac{h_{I}}{\eta}\right)^{\beta-1} \exp \left[-\left(\frac{h_{I}}{\eta}\right)^{\beta}\right]\left\{1-\exp \left[-\left(\frac{h_{I}}{\eta}\right)^{\beta}\right]\right\}^{\alpha-1},
$$

where $\beta>0$ and $\alpha>0$ are the shape parameters, and $\eta>0$ is the scale parameter. The parameter $\alpha, \beta$ depends on the scintillation index of the irradiance [13, Eq. 20,21], and the parameter $\eta$ depends on the mean value of the irradiance. The shape parameter $\alpha$ not characterizes the fading conditions of the link but also it along with parameter $\beta$ captures the effect of aperture averaging. [13].

Assuming a Gaussian spatial intensity profile for the beam waist, the misalignment fading $h_{m}$ is statistically characterized in [14]. For independent but identical Gaussian distributed horizontal sway and elevation, the radial displacement at the receiver follows a Rayleigh distribution. Now using these facts the PDF of $h_{m}$ is given, as in [12], as

$$
f_{h_{m}}\left(h_{m}\right)=\frac{\rho^{2}}{A_{0}^{2}} h_{m}^{\rho^{2}-1}, \quad 0 \leq h_{m} \leq A_{0}
$$

where $A_{0}$ is the fraction of the collected optical power and $\rho=\frac{\omega}{2 \sigma_{s}}, \omega$ is the equivalent beam width at the receiver, and $\sigma_{s}^{2}$ is the variance of pointing error displacement characterized by the horizontal sway and elevation.

For the path loss factor we consider the Kim's model proposed in [15]. The deterministic path loss factor for a channel of length $L$ is given by $h_{\ell}=\exp (-\mathcal{A} L)$, here $\mathcal{A}$ is the attenuation constant defined as

$$
\mathcal{A}=\frac{13}{V}\left[\frac{\lambda \times 10^{9}}{550}\right]^{\psi(V)}
$$

where $V$ is the visibility, $\lambda$ is operating wavelength, and $\psi(V)$ is the coefficient related to the size of atmospheric particles given by

$$
\psi(V)= \begin{cases}1.6 & \text { for } V>50 \mathrm{~km} \\ 1.3 & \text { for } 6<V<50 \mathrm{~km} \\ 0.16 V+0.34 & \text { for } 1<V<6 \mathrm{~km} \\ V-0.5 & \text { for } 0.5<V<1 \mathrm{~km} \\ 0 & \text { for } V<0.5 \mathrm{~km}\end{cases}
$$

\section{StATISTICS OF THE INSTANTANEOUS SNR}

In this section we derive the PDF and CDF of the instantaneous received SNR in the receiver. For a subcarrier intensity modulated communication, the SNR over channel $h$ is given by $\gamma=\bar{\gamma}_{0}|h|^{2}$, where $\bar{\gamma}_{0}=\frac{\left(P_{t} \mathcal{R} \zeta\right)^{2}}{N_{0}}$. The term $P_{t}$ is the average transmitter power, $\mathcal{R}$ is the responsitivity of the photodetector, $N_{0}$ is the average noise power of the additive white Gaussian noise at the receiver, and $\zeta$ is the modulation index [8, Eq. 3]. The average SNR can be given as $\bar{\gamma}=\bar{\gamma}_{0} E\left[|h|^{2}\right]$, where $E[\cdot]$ is the expectation operator [16].

Lemma 1: The PDF of the instantaneous SNR $\gamma$ can be given as

$$
f_{\gamma}(\gamma)=B_{1} \sum_{j=0}^{\infty} \Psi(j) \gamma^{\frac{\rho^{2}}{2}-1} \Gamma\left[\tau, B_{2}(j) \gamma^{\frac{\beta}{2}}\right],
$$

where $\Psi(j)=\frac{(-1)^{j} \Gamma(\alpha)}{j ! \Gamma(\alpha-j)(1+j)^{1-\frac{\rho^{2}}{\beta}}}, B_{1}=\frac{\alpha \rho^{2}}{2\left(h_{\ell} \eta A_{0} \sqrt{\bar{\gamma}_{0}}\right)^{\rho^{2}}}$, $\tau=1-\frac{\rho^{2}}{\beta}$, and $B_{2}(j)=\frac{1+j}{\left(h_{\ell} \eta A_{0} \sqrt{\bar{\gamma}_{0}}\right)^{\beta}}, \Gamma(\cdot)$ is the gamma function, and $\Gamma(\cdot, \cdot)$ is the upper incomplete gamma function [17].

Proof: In (1), the terms $h_{I}$ and $h_{m}$ are random variables (RVs) whose PDFs are given in (2) and (3), respectively, and the term $h_{\ell}$ is deterministic. In order to obtain the PDF of $h$, first we derive the PDF of RV $X=h_{I} h_{m}$. The PDF of $X$ can be written as,

$$
f_{X}(x)=\int_{\frac{x}{A_{0}}}^{\infty} f_{h_{m}}\left(x \mid h_{I}\right) f_{h_{I}}\left(h_{I}\right) d h_{I} .
$$

Using (2) and (3), (7) can be written as,

$$
\begin{aligned}
f_{X}(x)=\frac{\rho^{2}}{A_{0}^{\rho^{2}}} x^{\rho^{2}-1} & \int_{\frac{x}{A_{0}}}^{\infty} h_{I}{ }^{-\rho^{2}} \frac{\alpha \beta}{\eta}\left(\frac{h_{I}}{\eta}\right)^{\beta-1} \exp \left[-\left(\frac{h_{I}}{\eta}\right)^{\beta}\right] \\
& \times \underbrace{\left\{1-\exp \left[-\left(\frac{h_{I}}{\eta}\right)^{\beta}\right]\right\}^{\alpha-1} d h_{I} .}_{A 1}
\end{aligned}
$$

To solve the integration in (8), we expand the $A 1$ using the Newton's generalized binomial theorem i.e. $(1+y)^{t}=$ $\sum_{j=0}^{\infty} \frac{\Gamma(t+1) y^{j}}{\Gamma(t-j+1) j !}$. After some mathematical manipulations (8) can be rewritten as,

$$
\begin{aligned}
f_{X}(x) & =\frac{\alpha \beta \rho^{2}}{\eta^{\beta} A_{0}^{\rho^{2}}} x^{\rho^{2}-1} \sum_{j=0}^{\infty} \frac{(-1)^{j} \Gamma(\alpha)}{j ! \Gamma(\alpha-j)} \\
& \times \int_{\frac{x}{A_{0}}}^{\infty} h_{I}^{\beta-1-\rho^{2}} \exp \left(\frac{-(j+1)}{\eta^{\beta}} h_{I}^{\beta}\right) d h_{I} .
\end{aligned}
$$

The integral term in (9) can be solved using [17, Eq. 381.3], as

$$
f_{X}(x)=\frac{\alpha \rho^{2}}{\left(\eta A_{0}\right)^{\rho^{2}}} x^{\rho^{2}-1} \sum_{j=0}^{\infty} \Psi(j) \Gamma\left[1-\frac{\rho^{2}}{\beta}, \frac{(1+j) x^{\beta}}{\left(\eta A_{0}\right)^{\beta}}\right](10)
$$


The PDF of the channel coefficient $h=X h_{\ell}$ can be obtained as 1

$f_{h}(h)=\frac{\alpha \rho^{2}}{\left(h_{\ell} \eta A_{0}\right)^{\rho^{2}}} \sum_{j=0}^{\infty} \Psi(j) h^{\rho^{2}-1} \Gamma\left[1-\frac{\rho^{2}}{\beta}, \frac{1+j}{\left(h_{\ell} \eta A_{0}\right)^{\beta}} h^{\beta}\right], h \geq 0$.

Now the PDF of instantaneous SNR $\gamma$ defined can easily be derived using [16, Eq. 5-8].

Observation 1: The expression of the PDF in (6) consists of infinite summation which results from the use of Newton's generalized binomial expansion, however the for the given values of the parameters $\alpha, \beta$, and $\eta$, the infinite summation is convergent and ten to fifteen terms are sufficient for this series to converge [10] and can be verified analytically using MATLAB or MATHEMATICA. Further this can also be verified from Fig. 1, where we plotted the PDF for $j=0$ to 10 , and $j=0$ to 100 terms, and it can be observed that the plots of the PDF in both the cases are same.

Observation 2: The effect of channel parameters $\alpha, \beta$, and $\eta$ on the SNR can also be observed from Fig. 1. The increase in the parameters $\alpha$ and $\beta$ spreads the distribution as they are shape parameters and they control the steepness of the tail of the PDF. On the other hand, the height of the distribution reduces with an increase in the value of the parameter $\eta$ because it is the scale parameter and it determines the scale or the height of the distribution. Thus any change in the channel parameters affects the tail probabilities which are of maximum importance in the analysis of any communication system as the tail of distribution defines the error performance significantly.

Observation 3: To analyse the effect of $\bar{\gamma}_{0}$, we plotted the PDF for different values of average $S N R \quad \bar{\gamma}_{0}$ in Fig. 2. The plots suggest that the higher the $\bar{\gamma}_{0}$, wider will be the distribution and accordingly its height is scaled down.

Lemma 2: The CDF of the instantaneous SNR $\gamma$ can be given as

$$
F_{\gamma}(z)=\frac{2 B_{1}}{\beta} \sum_{j=0}^{\infty} \Psi(j) z^{\frac{\rho^{2}}{2}} G_{2,3}^{2,1}\left(\begin{array}{l|l}
B_{2}(j) z^{\frac{\beta}{2}} & \begin{array}{c}
1-\frac{\rho^{2}}{\beta}, 1 \\
0, \tau,-\frac{\rho^{2}}{\beta}
\end{array}
\end{array}\right),
$$

where $G_{p q}^{m, n}\left(\left.\cdot\right|^{\cdot}\right)$ is the Meijer's $\mathrm{G}$ function [18, Eq. (07.34.02.0001.01)].

Proof: The CDF of the SNR is defined as,

$$
\begin{aligned}
F_{\gamma}(z) & =\int_{0}^{z} f_{\gamma}(\gamma) d \gamma \\
& =B_{1} \sum_{j=0}^{\infty} \Psi(j) \int_{0}^{z} \gamma^{\frac{\rho^{2}}{2}-1} \Gamma\left[\tau, B_{2}(j) \gamma^{\frac{\beta}{2}}\right] .
\end{aligned}
$$

On replacing the upper incomplete gamma function in (13) with its Meijers-G equivalent [18, Eq. (06.06.26.0005.01)] and substituting $\gamma^{\frac{\beta}{2}}=t$ we get,

$$
F_{\gamma}(z)=\frac{2 B_{1}}{\beta} \sum_{j=0}^{\infty} \Psi(j) \int_{0}^{z^{\frac{\beta}{2}}} t^{\frac{\rho^{2}}{\beta}-1} G_{1,2}^{2,0}\left(B_{2}(j) t \mid \begin{array}{c}
1 \\
0, \tau
\end{array}\right) d t(14
$$

\footnotetext{
${ }^{1}$ The derived PDF $f_{h}(h)$ in (11) is a valid PDF as it is non-negative (see Table I), and area under this PDF is unity (see appendix for proof).
}

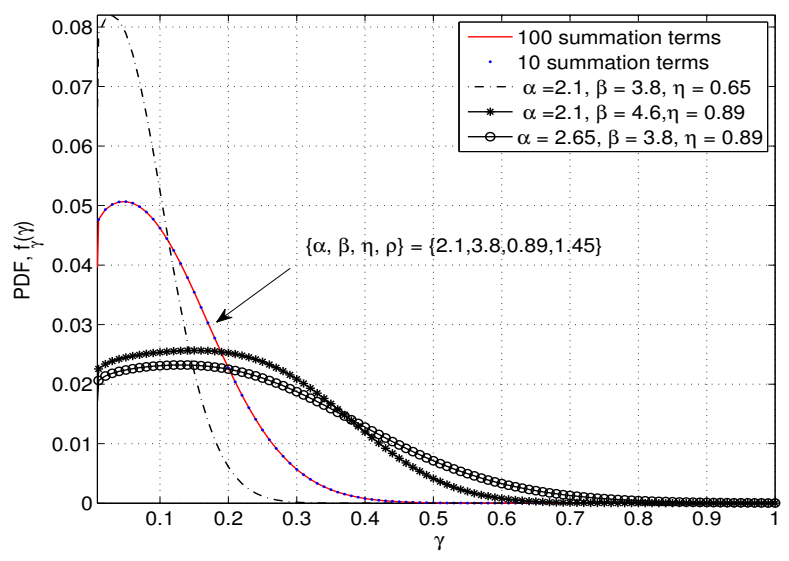

Fig. 1. The PDF of the instatntaneous SNR $\gamma$ for aperture-averaged receivers under the influence of atmospheric turbulence induced fading, misalignment fading and path loss.

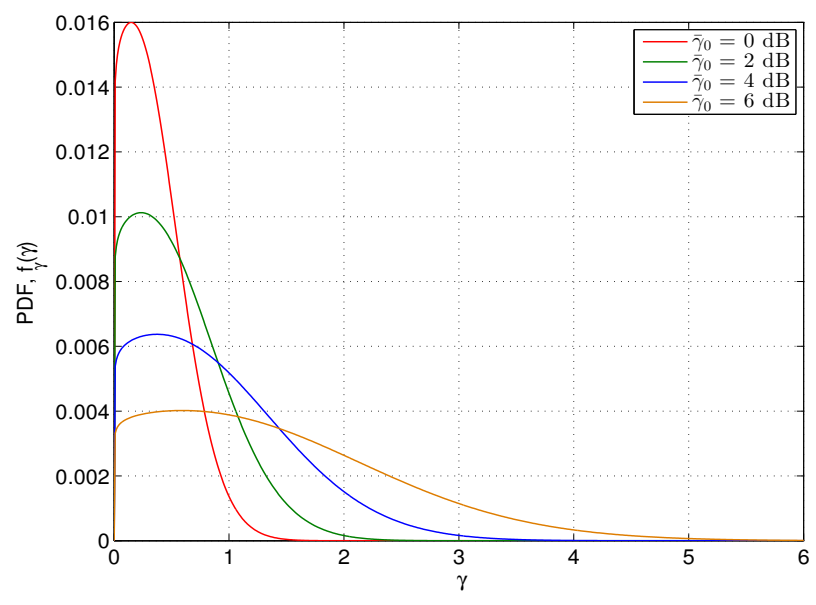

Fig. 2. The effect of average SNR $\bar{\gamma}_{0}$ on the PDF of the instantaneous SNR $\gamma$

This integral can be solved using [18, Eq. (07.34.21.0084.01)].

\section{PERFORMANCE ANALYSIS}

In this section we apply the derived statistics of the SNR to analyse the performance of an FSO communication system model. We consider a source and an aperture-averaged destination. The source has one transmit antenna aperture to send its message, and the destination has a finite sized receive antenna aperture for receiving from the source. The channel between the source and the destination is represented by the coefficient $h$ and is defined in (1). In the following subsections we will derive the expressions of the outage probability and the average BER.

\section{A. Outage Probability}

The outage probability $P_{\text {out }}$ is defined as the probability of instantaneous SNR $\gamma$ over a channel $h$ falling below a threshold value. We assume $\gamma_{t h}$ to be the threshold SNR, so the corresponding outage probability is,

$$
P_{\text {out }}=\operatorname{Pr}\left\{\gamma<\gamma_{t h}\right\}=\int_{0}^{\gamma_{t h}} f_{\gamma}(\gamma) d \gamma
$$




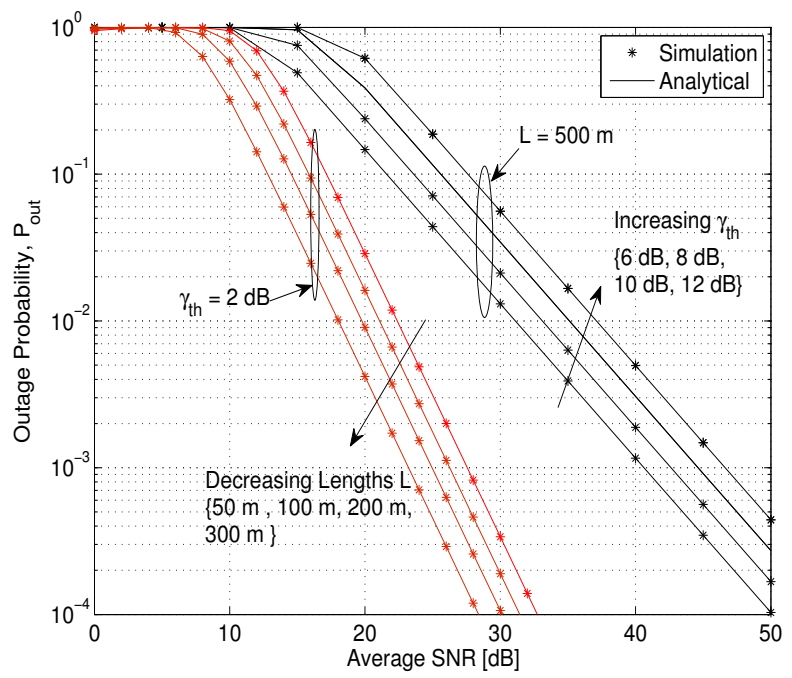

Fig. 3. Outage Probability vs av. SNR for different values of threshold SNR $\gamma_{t h}$, and channel length $L$.

The outage probability $P_{\text {out }}$ in (15) can be obtained using (12) as $P_{\text {out }}=F_{\gamma}\left(\gamma_{t h}\right)$.

\section{B. Average BER}

The average BER for binary modulation schemes can be given as [19]

$$
P(e)=\int_{0}^{\infty} P(e \mid \gamma) f_{\gamma}(\gamma) d \gamma
$$

where $P(e \mid \gamma)$ is the conditional error probability and for the given $\gamma$ it can be written as $P(e \mid \gamma)=\frac{\Gamma\left(\mu_{1}, \mu_{2} \gamma\right)}{2 \Gamma\left(\mu_{1}\right)}$, the parameters $\mu_{1}, \mu_{2}$ are modulation dependent constants and their values are : $\mu_{1}=\mu_{2}=0.5$ for coherent BPSK, $\mu_{1}=$ $0.5, \mu_{2}=1$ for coherent BFSK, $\mu_{1}=1, \mu_{2}=0.5$ for noncoherent BFSK, and $\mu_{1}=\mu_{2}=1$ for differential BPSK.

Now using [18, Eq. (06.06.26.0005.01)], and (6), followed by using [18, Eq. (07.34.21.0013.01)], the average BER in (16) can be obtained as,

$$
\begin{aligned}
P(e) & =\sum_{j=0}^{\infty} \frac{B_{1} k^{\tau-1 / 2} l^{\mu_{1}+\left(\rho^{2}-3\right) / 2} \mu_{2}^{-\rho^{2} / 2}}{2 \Gamma\left(\mu_{1}\right)(2 \pi)^{l+k-2}} \\
& \times G_{2 l+k, 2 k+l}^{2 k, 2 l}\left(\Omega(j) \mid \begin{array}{c}
\Delta(k, 1), \Delta(l, \varepsilon), \Delta\left(l, \varepsilon-\mu_{1}\right) \\
\Delta(k, 0), \Delta(k, \tau), \Delta(l, \varepsilon)
\end{array}\right)(17)
\end{aligned}
$$

where $l$ and $k$ are integer constants so that $\beta=\frac{l}{k}, \Delta(m, n)=$ $(n / m)((n+1) / m) \ldots .((n+m-1) / m), \Omega(j)=\frac{\left(B_{2}(j)\right)^{k} l^{l}}{\mu_{2} k^{k}}$ and $\varepsilon=1-\frac{\rho^{2}}{2}$.

The expressions of the average BER for various modulation schemes can be found on substituting the values of parameters $\mu_{1}$ and $\mu_{2}$ accordingly.

\section{Numerical RESUlts}

In this section, numerical results are presented for the outage performance of the considered system. In Fig. 3, the outage probability is plotted for various values of threshold SNR $\gamma_{t h}$, and link length $L$ considering $A_{0}=0.35, \rho=1$, $V=1.2 \mathrm{~km}, \alpha=2.18, \beta=4.0, \eta=0.5$. For various

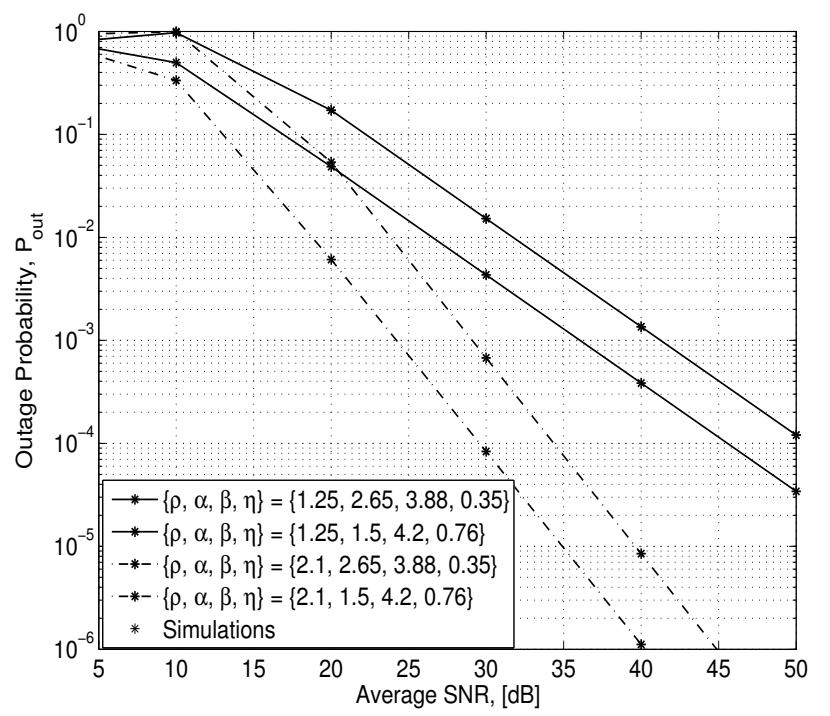

Fig. 4. Effect of misalignment fading : Outage probability vs av. SNR for different values of parameters $\{\alpha, \beta, \eta\}$.

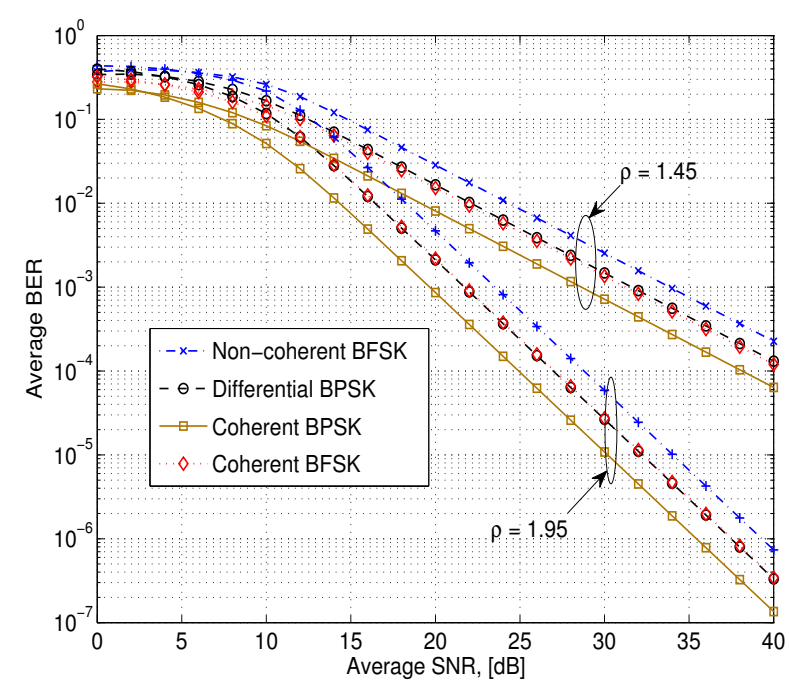

Fig. 5. Average BER for the different binary modulation schemes.

values of threshold SNR plots the link length is considered to be $L=500 \mathrm{~m}$, and for various values of link length plots the threshold SNR is taken as $\gamma_{t h}=2 \mathrm{~dB}$. It can be observed that the outage performance of the system deteriorates as the value of the target or threshold SNR increases. Further, as the separation between transmitter and receiver is increased, the outage probability increases and hence the system performance worsens, intuitively.

In Fig. 4, the effect of misalignment errors is observed on the outage performance with $A_{0}=0.85, \gamma_{t h}=5 \mathrm{~dB}$, $V=1.2 \mathrm{~km}, L=500 \mathrm{~m}$. In this figure the effects of the variations in the values of the parameters $\alpha, \beta$, and $\eta$ can be observed. Further, it can be seen from this figure that as the value of $\rho$ increases the outage probability decreases. This happens due the parameter $\rho$ is inversely proportional to the pointing error jitter at the receiver side [14], thus increased 
value of the parameter $\rho$ signifies the reduced level of the magnitude of the misalignment error.

Fig. 5 analytically plots the average BER of the various modulation schemes with respect to average SNR for different values of pointing error parameter $\rho$. The coherent BFSK, non-coherent BFSK, coherent BPSK, and Differential BPSK modulation schemes are considered. The values of other parameters are assumed as follows: $A_{0}=0.35, V=1.2$ $\mathrm{km}, \alpha=2.18, \beta=3.8, \eta=0.75$. The main observation from this plot is that for the given value of $\rho$ the average BER for coherent BFSK and differential BPSK is almost same. Further, the complexity of the coherent detection can be justified as the coherent BPSK outperforms the other detection techniques and provides the minimum gain of approximately $2.5 \mathrm{~dB}$, when compared to differential BPSK, and $4 \mathrm{~dB}$ when compared to non-coherent BFSK.

\section{CONCLUSIONS}

In this paper, a finite sized receiver aperture based communication system is analyzed over EW distributed FSO channels. Initially, the PDF of the composite channel coefficient has been derived with turbulence induced fading, distance dependent path loss, and misalignment fading. Using the PDF of the composite channel coefficient the statistics of the SNR over EW distribution were obtained. Finally, new closed form expressions for the outage probability and average BER were derived.

\section{APPENDIX}

TABLE I

\begin{tabular}{|l|l|l|l|l|l|l|l|}
\hline$h$ & 0 & 0.1 & 0.2 & 0.3 & 0.4 & 0.5 & 0.6 \\
\hline$f_{h}(h)$ & 0 & .0284 & .0609 & .0948 & .1279 & .1547 & .1656 \\
\hline$h$ & 0.7 & 0.8 & 0.9 & 1.0 & 1.1 & 1.2 & 1.3 \\
\hline$f_{h}(h)$ & .1513 & .1124 & .0645 & .0273 & .0082 & .0017 & .002 \\
\hline
\end{tabular}

For the expression in (11) to be a valid PDF it must be non-negative and area under this PDF should be unity. The non-negativity of PDF $f_{h}(h)$ in (11) can be observed from Table I as the range of $f_{h}(h)$ in the possible domain of $h$ is positive. Following is the proof of unit area under PDF $f_{h}(h)$ :

As the PDF given in (2) is a valid PDF [6], area under this PDF should be one i.e. $\int_{0}^{\infty} f_{h_{I}}\left(h_{I}\right) d h_{I}=1$. Rewriting the PDF $f_{h_{I}}\left(h_{I}\right)$ using Newton's generalized binomial theorem, we get

$$
\frac{\alpha \beta}{\eta} \sum_{j=0}^{\infty} \frac{(-1)^{j} \Gamma(\alpha)}{j ! \Gamma(\alpha-j)} \int_{0}^{\infty}\left(\frac{h_{I}}{\eta}\right)^{\beta-1} \exp \left[-(1+j)\left(\frac{h_{I}}{\eta}\right)^{\beta}\right] d h_{I}=1 .
$$

After solving this integral, followed by some mathematical rearrangements (18) reduces to

$$
\sum_{j=0}^{\infty} \frac{(-1)^{j} \Gamma(\alpha)}{j ! \Gamma(\alpha-j)(1+j)}=\frac{1}{\alpha} .
$$

The area under the PDF $f_{h}(h)$ is $\mathcal{S}=\int_{0}^{\infty} f_{h}(h) d h$. Using (11) we get,

$$
\mathcal{S}=\frac{\alpha \rho^{2}}{\left(h_{\ell} \eta A_{0}\right)^{\rho^{2}}} \sum_{j=0}^{\infty} \Psi(j) \int_{0}^{\infty} h^{\rho^{2}-1} \Gamma\left[1-\frac{\rho^{2}}{\beta}, \frac{1+j}{\left(h_{\ell} \eta A_{0}\right)^{\beta}} h^{\beta}\right] d h .
$$

On substituting $h^{\beta}=r$ in (20), and using [17, Eq. (6.455.1)], we get

$$
\mathcal{S}=\sum_{j=0}^{\infty} \frac{\Psi(j)}{(1+j)^{\frac{\rho^{2}}{\beta}}}{ }_{2} F_{1}\left(1,1 ; \frac{\rho^{2}}{\beta}+1 ; 0\right),
$$

where ${ }_{2} F_{1}(\cdot, \cdot ; \cdot ; \cdot)$ is Gauss hypergeometric function [18, Eq. (07.23.02.0001.01)]. Now using [18, Eq. (07.23.03.0001.01)] and (19) we get $\mathcal{S}=1$.

\section{REFERENCES}

[1] L.C. Andrews, R.L. Phillips, and C.Y. Hopen, "Laser beam scintillation with applications," Bellingham, Washington: SPIE Press, 20011.

[2] L.C. Andrews, and R.L. Phillips, "Laser beam propagation through random media", 2nd ed. Bellingham, Washington: SPIE Press, 2005.

[3] F. Xu, M.A. Khalighi, P. Caussé, and S. Bourennane, "Channel coding and time-diversity for optical wireless links," Optics Express, vol. 17, no. 2, pp. 872-887, Jan. 2009.

[4] M.A. Khalighi, N. Schwartz, N. Aitamer, and S. Bourennane, "Fading reduction by aperture averaging and spatial diversity in optical wireless systems," IEEE/OSA Journal of Optical Communications and Networking, vol.1, no.6, pp. 580-593, Nov. 2009.

[5] B. Epple, "Simplified channel model for simulation of free-space optical communications", IEEE/OSA Journal of Optical Communications and Networking, vol.2, no.5, pp. 293-304, 2010.

[6] R. Barrios and F. Dios, "Exponentiated Weibull distribution family under aperture averaging for Gaussian beam waves," Optics Express vol. 20, issue 12, pp. 13055-13064, 2012.

[7] R. Barrios and F. Dios, "Reply to comment on The exponentiated Weibull distribution family under aperture averaging for Gaussian beam waves" Optics Express vol. 20, issue 12, pp. 20684-20687, 2012.

[8] P. Wang, L. Zhang, L. Guo, F. Huang, T. Shang, R. Wang, and Y. Yang, "Average BER of subcarrier intensity modulated free space optical systems over the exponentiated Weibull fading channels," Optics Express vol. 22, issue 17, pp. 20828-20841, 2014.

[9] M.M. Ahmed, and K.T. Ahmmed, "An expression for average bit error rate of free space optical links over Exponentiated Weibull atmospheric turbulence channel," in proc. 2014 International Conference on Informatics, Electronics \& Vision (ICIEV), pp.1-6, 23-24 May 2014.

[10] R. Barrios and F. Dios, "Probability of fade and BER performance of FSO links over the exponentiated Weibull fading channel under aperture averaging," in Unmanned/Unattended Sensors and Sensor Networks IX, ser. Proc. SPIE, vol. 8540, p. 85400D, 2012.

[11] X. Yi, Z. Liu, and P. Yue, "Average BER of free-space optical systems in turbulent atmosphere with exponentiated Weibull distribution," Optics Letters, vol. 37, no. 24, pp. 5142-5144, 2012.

[12] M. Cheng, Y. Zhang, J. Gao, F. Wang, and F. Zhao, "Average capacity for optical wireless communication systems over exponentiated Weibull distribution non-Kolmogorov turbulent channels," Applied Optics, vol. 53, issue 18, pp. 4011-4017, 2014.

[13] R. Barrios, F. Dios, "Exponentiated Weibull model for the irradiance probability density function of a laser beam propagating through atmospheric turbulence", Optics \& Laser Technology, Vol. 45, pp. 1320, February 2013.

[14] A. A. Farid, and S. Hranilovic, "Outage capacity optimization for freespace optical links with pointing errors," J. of Light. Tech., vol. 25, no. 7, pp. 1702-1710, July 2007.

[15] I. Kim, B. McArthur, and E. Korevaar, "Comparison of laser beam propagation at $785 \mathrm{~nm}$ and $1550 \mathrm{~nm}$ in fog and haze for optical wireless communications", Optical Wireless Communications III - Proceedings of SPIE vol. 4214, p. 26-37, Feb. 2001.

[16] A. Papoulis and S. U. Pillai, "Probability, random variables and stochastic processes",4th edition , Tata McGraw Hill, New Delhi, 2002.

[17] I. S. Gradshetyn and I. M. Ryzhic, Table of integrals, series and products, (U.S.A.: Academic Press, 6th ed.), 2000.

[18] I. Wolfram Research, Mathematica Edition: Version 8.0, Champaign, Illinois:Wolfram Research Inc., 2010.

[19] A. H. Wojnar, "Unknown bounds on performance in Nakagami channels," IEEE Transactions on Communications, vol. 34, no. 1, pp. 22-24, Jan. 1986. 